\title{
Exploration of the mechanism of Ziyin Tongluo Formula on preventing and treating postmenopausal osteoporosis based on the network pharmacology and molecular docking
}

\section{Rong-Bin Chen}

Guangdong Province Hospital of Traditional Chinese Medicine ZHUHAI BRANCH

\section{Ying-Dong Yang}

Guangzhou University of Chinese Medicine

\section{Kai Sun}

Guangzhou University of Chinese Medicine

\section{Shan Liu}

The Research Center of Basic Intergrative Medicine, Guangzhou University of Chinese Medicine, Guangzhou, China

\section{Wei Guo}

Guangzhou University of Chinese Medicine

\section{Jin-Xin Zhang}

Guangdong Province Hospital of Traditional Chinese Medicine ZHUHAI BRANCH

\section{Yong Li ( $\nabla$ yygctm@163.com )}

Guangdong Province Hospital of Traditional Chinese Medicine ZHUHAI BRANCH https://orcid.org/0000-0003-4257-032X

\section{Research}

Keywords: Postmenopausal osteoporosis, Ziyin Tongluo Formula, Traditional Chinese medicine, Network pharmacology, Molecular docking

Posted Date: October 27th, 2020

DOl: https://doi.org/10.21203/rs.3.rs-39351/v2

License: (c) (i) This work is licensed under a Creative Commons Attribution 4.0 International License. Read Full License 


\section{Abstract}

Background: Postmenopausal osteoporosis (PMOP) is a global chronic and metabolic bone disease, which poses huge challenges to individuals and society. Ziyin Tongluo Formula (ZYTLF) has been proved effective in the treatment of PMOP. However, the material basis and mechanism of ZYLTF against PMOP have not been thoroughly elucidated.

Methods: Online databases were used to identify the active ingredients of ZYTLF and corresponding putative targets. Genes associated with PMOP were mined, and then mapped with the putative targets to obtain overlapping genes. Multiple networks were constructed and analyzed, from which the key genes were selected. The key genes were imported to the DAVID database to performs GO and KEGG pathway enrichment analysis. Finally, AutoDock Tools and other software were used for molecular docking of core compounds and key proteins.

Results: Ninety-two active compounds of ZYTLF corresponded to 243 targets, with 129 target genes interacting with PMOP, and 50 key genes were selected. Network analysis showed the top 5 active ingredients including quercetin, kaempferol, luteolin, scutellarein, and formononetin., and the top 50 key genes such as VEGFA, MAPK8, AKT1, TNF, ESR1. Enrichment analysis uncovered two significant types of KEGG pathways in PMOP, hormone-related signaling pathways (estrogen , prolactin, and thyroid hormone signaling pathway) and inflammation-related pathways (TNF, PI3K-Akt, and MAPK signaling pathway). Moreover, molecular docking analysis verified that the main active compounds were tightly bound to the core proteins, further confirming the anti-PMOP effects.

Conclusions: Based on network pharmacology and molecular docking technology, this study initially revealed the mechanisms of ZYTLF on PMOP, which involves multiple targets and multiple pathways.

\section{Background}

Postmenopausal osteoporosis (PMOP) refers to a global chronic metabolic bone disease with increased fragility and fracture susceptibility occurring in postmenopausal women, who are confronted with the drop of ovarian function and estrogen levels. It is characterized by greater bone resorption than bone formation, low bone mass and bone microstructure destruction. Osteoporotic fractures are one of the main causes of post-menopausal women's disability and death. Therefore, PMOP has now become a major issue of life quality worldwide, and its prevention research is one of the hot spots in modern medical research [1-2]. The current drug treatment on PMOP can be divided into bone resorption inhibitors (estrogen, bisphosphonates, calcitonin), bone formation promoters (parathyroid hormone, fluoride), and bone mineralization (calcium, active Vitamin D). Estrogen replacement therapy has achieved good results in the clinic, but its cost is expensive, and the side effect such as endometrial hyperplasia, increased breast cancer risk [3], gastrointestinal reactions [4] are still one of the clinical problem.

Modern pharmacological experiments have confirmed that a variety of traditional Chinese herbs contain active ingredients for anti-osteoporosis, and formula of traditional Chinese herbs have stronger anti- 
osteoporosis effects than single of one [5-6]. Ziyin Tongluo Formula (ZYTLF) is a constitution of 14 traditional Chinese herbs, including Rehmannia glutinosa (Shudi), Ophiopogon japonicus (Maidong), Ligustrum Lucidum (Nuzhenzi), Angelica sinensis (Danggui), Paeonia albiflora (Baishao), Achyranthes bidentata (Niuxi), Loranthus parasiticus (Sangjisheng), Caulis Millettiae (Jixueteng), Zaocys (Wushaoshe), Scolopendra subspinipes mutilans (Wugong), Astragalus membranaceus (Huangqi), Saposhnikovia divaricata (Fangfeng), Atractylodes macrocephala (Baizhu), Radix Glycyrrhizae Preparata (Gancao). Previous papers have shown that ZYTLF can effectively alleviate the clinical symptoms and increase bone density of patients with PMOP [7], but its molecular mechanism of action is still unclear.

Based on network pharmacology and molecular docking analysis, this study tries to screen the main active ingredients of ZYTLF in the prevention and treatment of PMOP, its targets and signaling pathways, and provides directions for elucidating the material basis and mechanism of action of ZYTLF in the prevention and treatment of PMOP. (Fig 1).

\section{Methods}

Identification of ZYTLF active ingredients

A total of 14 traditional Chinese herbs were imported into the Traditional Chinese Medicine Systems Pharmacology Database and Analysis Platform (TCMSP http://tcmspw.com/tcmsp.php) [8], BAT-MAN TCM (http://bionet.ncpsb.org/batman-tcm/) [9] and Traditional Chinese Medicine Information Database(TCMID, http://www.megabionet.org/tcmid/) [10] to obtain corresponding certain or potential compounds. The ADME (absorption, distribution, metabolism and excretion) properties of a compound is of importance in drug discovery and development [11]. Therefore, oral bioavailability (OB) and druglikeness (DL) , the two most important indicators of bioinformatics to evaluate the characteristics of ADME, were used as standards for screening active compounds. The TCMSP database utilizes Obioavail 1.1 to calculate the OB value [12], and applies the Tanimoto coefficient, see formula 1 in the

\section{supplementary files section.}

to calculate the $\mathrm{DL}$ value. Compounds that meet the requirement $\mathrm{OB} \geq 30 \%$ and $\mathrm{DL} \geq 0.18$ were regarded as biologically active ingredients. Because Radix Glycyrrhizae Preparata is less important in this formula, the standard was set as $\mathrm{OB} \geq 60 \%$ and $\mathrm{DL} \geq 0.36$ to avoid greater bias. Based on the above conditions, the active ingredients of ZYTLF can be screened by TCMSP for subsequent analysis.

\section{Prediction of ZYTLF targets}

The next step after screening active ingredients was to predict the targets that might stimulate biological effects. The TCMSP analysis platform is loaded with prediction function of large-scale molecular network targets based on chemistry, genomics, pharmacology and system analysis technology, which is currently a commonly used target prediction platform [12]. After inputting the active ingredient into the TCMSP analysis platform, the corresponding target could be collected. Uniprot (https: //www.Uniprot.org) [13] is a database containing accurate annotations of proteins and other substances, where the genetic 
information of the target can be obtained. The target information were inputted into the Uniprot database to acquire the standard gene name.

Acquisition of PMOP-related genes and overlapping genes

First of all, Osteoporosis and Postmenopausal were used as the key words to collect PMOP related genes from the three following online databases, Genecards (http://www.genecards.org) [14], OMIM (http://omim.org/) [15], and DisGenet (https://www.disgenet.org/) [16]. Next, overlapping target genes were obtained from ZYTLF target genes and PMOP-related genes, which may be potential targets for ZYTLF against PMOP.

Construction of a network model of "herb-active ingredient-overlapping gene"

The overlapping target genes and their corresponding active ingredients and herbs were inputted into Cytoscape 3.7.1 software [17], to build a "herb-active ingredient-overlapping gene" network model. CytoNCA plug-in [18] was used to conduct network topology analysis. According to the Degree Centrality (DC) and Between Centrality (BC), the key nodes in the network were screened. The higher the node's degree value was, the more important it was in the network.

\section{Construction of PPI network of overlapping genes}

The STRING database (http://string-db.org/, ver.11.0) [19] was utilized to constructed protein-protein interaction (PPI) network of overlapping proteins, setting the condition of data analysis mode "Multiple proteins", the type "Homo sapiens" (human), and the minimum mutual threshold "high confidence $(0.700)$ ". The remaining parameters remained unchanged. The information of PPI network was obtained and then imported into Cytoscape 3.7.1 software. The MCC algorithm in the CytoHubba plug-in was applied to perform network analysis to screen out core genes.

Enrichment analysis of core genes

The Database Visualization and Integrated Discovery system (DAVID, https://david.ncifcrf.gov/) is an online biological information database, integrating biological data and analysis tools to provide annotation information of comprehensive biological function. Quantitative screening technology has its advantages on the biological function analysis of genes [20-21]. The species was limited to "Homo sapiens", and enrichment analysis of Gene Ontology (GO, http: //www.geneontology.org/) and Kyoto Encyclopedia of Genes and Genomes (KEGG, http: //www .genome.jp/kegg/) was conducted. R software was used to draw advanced bubble diagrams and gene-pathway mechanism diagrams was obtained from the KEGG database.

Molecular docking simulation

The computer simulation docking technology was utilized to verify the reliability of this study through evaluating the binding efficiency of the overlapping proteins and main active ingredients in ZYTLF. To 
obtain the SDF structure of top 10 core compounds in the network from the PubChem database (https://pubchem. ncbi.nlm.nih.gov/). The 2D structure was processed and transformed into PDB format through PyMOL, and they were saved in PDBQT format as docking ligands. Simultaneously, to collect all the crystal structures of the key proteins from the RCSB protein data bank (PDB, http://www.pdb.org/) and select the ones with relatively higher resolution and unique ligands. AutoDock Tools software was used to remove the water molecules, isolate proteins and save it as receptors. The receptors and ligands were processed with PyMOL software and Auto Dock software and then docked through Vina.

\section{Results}

Active ingredients and corresponding targets of ZYTLF

Through TSMSP platform, BAT-MAN TCM and TCMID database, a total of 1,738 compounds were obtained, and 92 compounds that met the standard were regarded as active ingredients. The detailed information of active ingredients of ZYTLF are displayed in Additional file 1:Table S1. There were 242 corresponding target genes for these active ingredients.

PMOP-related genes and overlapping genes

From Genecard, Disgenet and OMIM databases, a total of 1113 PMOP related genes after removing duplication were obtained. Compared target genes of ZYTLF with PMOP-related genes, a total of 129 overlapping target genes were acquired. (Fig. 2).The detailed information of ZYTLF-genes, PMOP-genes, and ZYTLF-PMOP overlapping target genes are shown in Additional file 2:Table S2.

Network analysis

A network of "herb-active ingredient-overlapping gene" was constructed. (Fig. 3). There were 231 nodes and 1,072 edges in the network, and the detailed information of the network are shown in Additional file 3:Table S3. The results of network topology analysis showed that the active ingredients such as quercetin, kaempferol, luteolin, scutellarein, and formononetin had higher degrees, which played an important role in the network. The network revealed that one active compound could correspond with multiple genes, and one gene could also correspond with multiple active compounds. The complex interaction between active components and intersecting genes manifested the multi-component and multi-target features of ZYTLF.

The intersecting genes were inputted into the STRING database to built a PPI network after setting the relevant parameters. The network displayed the relevant information of the genes and the interaction relationship. (Fig. 4). Then Cytoscape 3.7.1 software was used to visually process and analyze the PPI network information. Based on the MCC algorithm of the CytoHubba plug-in, the top 50 key genes were selected. (Fig. 5).

GO and KEGG enrichment analysis 
DAVID database was used to perform GO enrichment and KEGG pathway enrichment analysis on 50 core genes and extract significant enrichment results (FDR < 0.05). A total of $62 \mathrm{GO}-\mathrm{BP}$ terms, $6 \mathrm{GO}-\mathrm{CC}$ terms, 11 GO-MF terms and 59 terms on the KEEG pathway were obtained. The enrichment results showed that the biological processes involved mainly included positive transcriptional regulation signals of RNA polymerase II promoter, negative regulation of apoptosis, positive regulation of transcription using DNA as a template, aging, positive regulation of gene expression, etc.. Main cell components included nucleus, cytoplasm, extracellular space, etc. Regulated molecular functions mainly included enzyme binding, transcription factor binding, and protein binding. (Fig. 6 a-c).

A total of 15 signal pathways related to PMOP were screened from the significant signal pathways. (Fig. $6 \mathrm{~d})$. The signal pathways mainly involved inflammation, including TNF, HIF-1, PI3K-Akt, Toll-like receptors, MAPK and other signaling pathways, and hormonal pathways such as estrogen, prolactin, and thyroid hormone signaling pathways, indicating ZYTLF could play a synergistic role through many pathways. From the KEGG database, the mechanism of action of key genes on estrogen and TNF signaling pathways was obtained. The red marks in the figure represent potential targets for possible intervention by ZYTLF. (Fig. 7 and Fig. 8). The results and detailed information on the GO terms and pathways are shown in Additional file 4:Table S4.

The core compounds in ZYTLF are docked with key proteins

Molecular docking simulation was applied to verify if the top 10 compounds have an pivotal role in regulation of the 50 key proteins. In molecular docking, if the ligand can bind to one or more amino acid residues in the active site (also called active pocket) of the receptor and participates in the process of conformation change, energy complementation, and so on, then the small molecule ligand can combine with the receptor to form a stable structure. This study indicated that the top 10 compounds had strong affinity with 23 core proteins,including ESR1 (PDB id: 4pxm), TNF (PDB id: 3m2w), AKT1 (PDB id: 6npz), PTGS2 (PDB id: 5f19), which have an important role according to the result of network pharmacology.

The quercetin can bind closely with the active pocket of ESR1 and TNF proteins. Quercetin developed four hydrogen bonds with the amino acid residues LEU-72, LYS-93, EU-141, VAL-78, making quercetin and TNF form a stable complex(Fig. 9). Meanwhile, Quercetin developed three hydrogen bonds with the amino acid residues GLY-521, GLU-353, ARG-394, making quercetin and ESR1 form a stable complex(Fig. 10). The binding energies of the various compounds are shown in Additional file 5:Table S5.

\section{Discussion}

At the molecular level, multiple factors participate in the development of PMOP, interacting with each other and regulating bone metabolism in a complex regulatory network. Estrogen deficiency is the pivotal factor in the development of PMOP, which causes to the disorder of bone metabolism. Bone resorption is greater than bone formation, and eventually osteoporosis is caused [22]. Compared with hormone replacement therapy, traditional Chinese medicine, as a welcome alternative therapy, has greater advantages possessing many merits of simplicity and low side effect. Modern research have found that 
traditional Chinese herb contains various bioactive ingredients, which can act on multiple targets through multiple pathways, validating the holistic view of traditional Chinese medicine and the theory of treatment based on syndrome differentiation [23]. Compared with the "single component-single targetsingle pathway" research model of traditional pharmacology, network pharmacology combines the concepts of bioinformatics and multi-directional pharmacology to analyze the relationship between biological systems, medicines, and diseases from a network perspective. It has opened a new research model of traditional Chinese medicine from empirical medicine to evidence-based medicine [24].

\section{Key active ingredient of ZYTLF for anti-PMOP}

According to the network analysis results of the "herb-active ingredient-overlapping gene", the key active ingredients of ZYTLF are quercetin, kaempferol, luteolin, rhubarbin, formononetin and other flavonoid compounds. At the same time, they are also phytoestrogens, which has two-way regulating effects. It exerts the role of estrogen on target organ when estrogen deficiency occurs in body, and plays the role of anti-estrogen when the level of estrogen increases. It can alleviate a series of clinical symptoms caused by the postmenopausal estrogen deficiency and avoid the occurrence of tumors in the reproductive system caused by estrogen replacement therapy [25]. Quercetin with the highest degree value in the network is a typical flavonoid compound. It has multiple pharmacological effects, including scavenging free radical, anti-cancer, anti-infection, and protecting cardiovascular [26-29]. Quercetin has also been proven to be an effective component against osteoporosis, with the dual effects of inhibiting osteoclastogenesis and osteoblast differentiation. Zhixing Li found that quercetin can relieve osteoporosis symptoms in ovariectomized rats, possibly by up-regulating ALP gene expression and inhibiting JNK, ERK, and p38 MAPK signaling pathways [30]. Quercetin can also directly or indirectly down-regulate the expression of RANKL, inhibit osteoclast differentiation, reduce bone resorption, and stimulate osteoblast activity through estrogen signaling pathway and ERK signaling pathway [31]. Kaempferol has also been shown to have bone protective effects on ovariectomized rats [32], possibly through estrogen receptor, MAPK, NF-KB and other signaling pathways [33]. Luteolin can prevent bone loss after osteoporosis by inhibiting osteoclast differentiation.

\section{Key Genes of ZYTLF for Preventing PMOP}

Combined with the analysis of PPI network and key gene network, TNF, JUN, MAPK8, AKT1, IL-6, MMP9, PTGS2, MAPK1, CASP3 were of great importance and all related to the inflammatory response process. Actually, increasing studies have found that the reduction of estrogen levels in postmenopausal women can stimulate the immune system to produce a large number of osteoclastogenic factors, which in turn activates related signaling pathways, further aggravating bone loss [33]. Zha Li and other scholars have found that postmenopausal women with osteoporosis have significantly increased levels of TNF-a. Invitro experiments shows that TNF- $a$ and RANKL synergistically enhance bone resorption of osteoclasts through NF-KB and PI3K/Akt signaling pathways [35]. TNF- $\alpha$ and IL6 play an important role in the immune response and bone metabolism, mainly affecting the differentiation and proliferation of osteoclasts by regulating complex mechanisms, and they are important pathogenic factors for immune- 
mediated bone diseases [36]. Studies have shown that increased levels of inflammatory factor TNF-a in the serum of postmenopausal women may be one of the important causes of osteoporosis. TNF- $a$ can activate the RANK/RANKL signaling pathway and induce the formation of osteoclasts [37]. We speculates that elevated TNF-a level in the serum of postmenopausal women with osteoporosis may be related to estrogen deficiency.

The signal pathways ZYTLF in preventing PMOP

The enrichment analysis of KEGG pathways for the key genes of ZYTLF in preventing PMOP showed that 15 signaling pathways were related to the development and progression of PMOP, including hormone pathways and inflammation-related pathways. Hormones in postmenopausal women, including estrogen [38], parathyroid hormone [39], and prolactin, if not normally secreted could affect bone metabolism [40]. Estrogen bound with the estrogen receptor in osteoblasts and osteoclasts to act on the OPG/RANK/RANKL signaling pathway, which further promoted OPG secretion, down-regulated the expression of RANKL, and inhibited the formation of osteoclasts [41]. After the combination of estrogen and estrogen receptor, it could also regulate the expression of various target genes through the estrogen signaling pathway, thereby activating downstream PI3K/Akt, MAPK, WNT and other signaling pathways to promote the differentiation and proliferation of osteoblasts [42-43]. The TNF signaling pathway had the largest number of enriched genes, which might be the key signaling pathway for ZYTLF to prevent PMOP. The TNF signaling pathway was mainly opened by TNF- $a$ and interacted with multiple signaling pathways to synergistically inhibit osteoclast differentiation and bone resorption function [44].

Verification of molecular docking

In this study, network pharmacology was applied to elaborate on the potential anti-PMOP effect of ZYTLF and this effect was visualized by molecular docking for verification. In the result, ESR, TNF and other 21 proteins can combined with the top 10 active compounds, which have a significant role in regulation of the 23 proteins associated closely with PMOP. The result proved that ZYTLF could exert the treatment of PMOP by activating those receptors in the estrogen signaling pathway and TNF signaling pathway. It is consistent with the result of a vitro study showing that quercetin protected against TNF-a induced impairments in bone marrow mesenchymal stem cells[45]. In another study, quercetin can exert dual effect on estrogen, displaying anti-estrogenic effect at low dose $(10 \mathrm{mg} / \mathrm{kg} / \mathrm{day})$, and exaggerated estrogenic activities at high dose $(100 \mathrm{mg} / \mathrm{kg} /$ day $)[46]$.

However, there are several limitations in this study. First, the compatibility and dosage of the formula are not taken into consideration. Second, the screened active components are different from the actual components in blood. Third, even if the results of network pharmacology and molecular docking simulation were combined, we still could not completely understand the accurate therapeutic mechanism of ZYTLF. Therefore, further experiments need to be conducted combining with Western Blot, Rt-PCR and multi-omics technology, etc. However, the network pharmacology methodology can save our time and energy by predicting the active ingredients, targets and signal pathways, and verifying it with molecular docking simulation. 


\section{Conclusions}

Quercetin, kaempferol, luteolin and other core compounds of ZYTLF may be enriched in estrogen signaling pathway and TNF signaling pathway through ESR1, TNF, IL6, MAPK8, STAT3, MMP9, PTGS2, JUN, inhibiting osteoclast formation, promoting osteoblast differentiation, regulating bone metabolism in the treatment of PMOP.

\section{Declarations}

Acknowledgements

Not applicable

Funding

This study was supported by grants from Traditional Chinese Medicine Bureau Of Guangdong Province for Arranged Project of Experiential prescription (No. 20194010). Funder provided financial support for the study.

Availability of data and materials

The datasets used and analyzed during the current study are available from the corresponding author on reasonable request.

Ethics approval and consent to participate

Not applicable

Competing interests

The authors declare that they have no competing interests.

Consent for publication

Not applicable

Authors' contributions

YL and RBC conceived and designed the study. RBC and YDY drafted the manuscript. JXZ and SL collected the data. YDY. KS and WG performed the data analysis. KS, SL, and WG provided advice during the study and manuscript preparation. All authors read and approved the final manuscript.

Author's information 
Rong-Bin Chen (469843897@qq.com), Ying-Dong Yang (125159770@qq.com), Kai Sun (961220881@qq.com), Shan Liu (liushan@gzucm.edu.cn),Wei Guo (844608579@qq.com), Jin-Xin Zhang (352339532@qq.com), Yong Li (lyzhuhai666@126.com)

Author details

${ }^{1}$ Guangzhou University of Chinese Medicine, Guangzhou 510006, China. ${ }^{2}$ GuangDong Province Hospital Of Traditional Chinese Medicine ZHUHAI BRANCH, Zhuhai 519015, China. ${ }^{3}$ The Research Center of Basic Integrative Medicine, Guangzhou University of Chinese Medicine, Guangzhou 510006, China.

\section{Abbreviations}

PMOP:postmenopausal osteoporosis; ZYTLF:Ziyin Tongluo Formula; TCMSP: traditional Chinese medicine system pharmacology analysis platform; GO: Gene Ontology; KEGG: Kyoto Encyclopedia of Genes and Genomes; BP: biological processes; CC: cellular components; MF: molecular functions; OB: oral bioavailability; DL: drug-likeness; ADME: absorption, distribution, metabolism, excretion; IL-6: Interleukin 6; TNF:Tumor necrosis factor.

\section{References}

1. Fujiwara S. Epidemiology of respiratory diseases and osteoporosis. Clin Calcium 2016;26 (10):13871392.Japanese. PMID: 27666684.

2. Eastell R, O'Neill TW, Hofbauer LC, Langdahl B, Reid IR, Gold DT, Cummings SR. Postmenopausal osteoporosis. NAT REV DIS PRIMERS 2016;2(1). https://doi.org/10.1038/nrdp.2016.69.

3. Tella SH, Gallagher JC. Prevention and treatment of postmenopausal osteoporosis. J Steroid Biochem Mol Biol 2014;142:155-70. https://doi.org/10.1016/j.jsbmb.2013.09.008.

4. Campbell IA, Douglas JG, Francis RM, Prescott RJ, Reid D M. Hormone replacement therapy (HRT) or etidronate for osteoporosis in postmenopausal asthmatics on glucocorticoids: a randomised factorial trial. Scott Med J 2009;54(1):21-5. https://doi.org/10.1258/rsmsmj.54.1.21.

5. Lin J, Zhu J, Wang Y, Zhang N, Gober HJ, Qiu XM, Li DJ, Wang L. Chinese single herbs and active ingredients for postmenopausal osteoporosis: From preclinical evidence to action mechanism. BIOSCI TRENDS 2017;11(5):496-506. https://doi.org/10.5582/bst.2017.01216.

6. Zhang ND, Han T, Huang BK, Rahman K, Jiang YP, Xu HT, Qin LP, Xin HL, Zhang QY, Li YM. Traditional Chinese medicine formulas for the treatment of osteoporosis: Implication for antiosteoporotic medicine discovery. J ETHNOPHARMACOL 2016;189:61-80. https://doi.org/10.1016/j.jep.2016.05.025.

7. Chen MS, Zhang B, Zeng HB, Tang SD. Clinical study on treating postmenopausal osteoporosis of the ShenyinXu type with the Ziyin Tongluo prescription. Clinical Journal of Chinese Medicine 2019;11(14):108-110.2019-05-20. 
8. 8. Ru J, Li P, Wang J, Zhou W, Li B, Huang C, Li P, Guo Z, Tao W, Yang Y, Xu X, Li Y, Wang Y, Yang L. TCMSP: a database of systems pharmacology for medicine discovery from herbal medicines. $J$ Cheminform 2014;6:13. https://doi.org/10.1186/1758-2946-6-13.

9. Liu Z, Guo F, Wang Y, Li C, Zhang X, Li H, Diao L, Gu J, Wang W, Li D, He F. BATMAN-TCM: a Bioinformatics Analysis Tool for Molecular mechANism of Traditional Chinese Medicine. Sci Rep. 2016, 6:21146. https://doi.org/10.1038/srep21146.

10. Lin Huang, Duoli Xie, Yiran Yu, Huanlong Liu, Yan Shi, Tieliu Shi, Chengping Wen. TCMID 2.0: a comprehensive resource for TCM. NUCLEIC ACIDS RES 2018;46(D1):D1117-D1120. https://doi.org/doi: 10.1093/nar/gkx1028.

11. Guan LF, Yang HB, Cai YC, SunLX, Di PW, Li WH, Liu GX, Tang Y. ADMET-score - a comprehensive scoring function for evaluation of chemical medicine-likeness. MEDCHEMCOMM 2019;10(1):148157. https://doi.org/10.1039/C8MD00472B.

12. Xu X, Zhang WX, Huang C, Li Y, Yu H, Wang YH, Duan JY, Ling Y. A Novel Chemometric Method for the Prediction of Human Oral Bioavailability. INT J MOL SCI 2012;13(6):6964-6982. https://doi.org/10.3390/ijms13066964.

13. UniProt: a worldwide hub of protein knowledge. NUCLEIC ACIDS RES 2019;47(D1):D506-D515. https://doi.org/10.1093/nar/gky1049.

14. Safran M. Human Gene-Centric Databases at the Weizmann Institute of Science: GeneCards, UDB, CroW 21 and HORDE. NUCLEIC ACIDS RES 2003;31(1):142-146. https://doi.org/10.1093/nar/gkg050.

15. Amberger JS, Hamosh A. Searching Online Mendelian Inheritance in Man (OMIM): A Knowledgebase of Human Genes and Genetic Phenotypes. Current Protocols in Bioinformatics 2017;58(1). https://doi.org/10.1002/cpbi.27.

16. Janet Piñero, Juan Manuel Ramírez-Anguita, Josep Saüch-Pitarch, Francesco Ronzano, Emilio Centeno, Ferran Sanz, Laura I. Furlong. The DisGeNET knowledge platform for disease genomics: 2019 update. NUCLEIC ACIDS RES 2019. https://doi.org/10.1093/nar/gkz1021.

17. Doncheva NT, Morris JH, Gorodkin J, Jensen LJ. Cytoscape StringApp: Network Analysis and Visualization of Proteomics Data. J PROTEOME RES 2018;18(2):623-632. https://doi.org/doi: 10.1021/acs.jproteome.8b00702.

18. Tang Y, Li M, Wang JX, Pan Y, Wu FX. CytoNCA: A cytoscape plugin for centrality analysis and evaluation of protein interaction networks. BIOSYSTEMS 2015;127:67-72. https://doi.org/doi: 10.1021/acs.jproteome.8b00702.

19. Szklarczyk D, Gable AL, Lyon D, Junge A, Wyder S, Huerta-Cepas J, Simonovic M, Doncheva NT, Morris JH, Bork P, Jensen LJ, Mering CV. STRING v11: protein-protein association networks with increased coverage, supporting functional discovery in genome-wide experimental datasets. NUCLEIC ACIDS RES 2019;47(D1):D607-D613. https://doi.org/10.1093/nar/gky1131.

20. Huang DW, Sherman BT, Lempicki RA. Systematic and integrative analysis of large gene lists using DAVID bioinformatics resources. NAT PROTOC 2009;4(1):44-57. 
https://doi.org/10.1038/nprot.2008.211.

21. Huang DW, Sherman BT, Lempicki RA. Bioinformatics enrichment tools: paths toward the comprehensive functional analysis of large gene lists. NUCLEIC ACIDS RES 2009;37(1):1-13. https://doi.org/10.1093/nar/gkn923.

22. Raisz LG. Pathogenesis of osteoporosis: concepts, conflicts, and prospects. J CLIN INVEST 2005;115(12):3318-3325. https://doi.org/10.1172/JCl270712005-12-01].

23. Zheng JH, Wu M, Wang HY, Li SS, Wang X, Li Y, Wang D, Li S. Network Pharmacology to Unveil the Biological Basis of Health-Strengthening Herbal Medicine in Cancer Treatment. CANCERS 2018;10(11):461. https://doi.org/10.3390/cancers10110461.

24. Zhang RZ, Zhu X, Bai H, Ning N. Network Pharmacology Databases for Traditional Chinese Medicine: Review and Assessment. FRONT PHARMACOL 2019;10. https://doi.org/10.3389/fphar.2019.00123.

25. Ludmila Křrižová, Kateřina Dadáková, Jitka Kašparovská, Tomáš Kašparovský. Isoflavones. MOLECULES 2019;24(6):1076. https://doi.org/10.3390/molecules24061076.

26. Ranganathan Babujanarthanam, Purushothaman Kavitha, U. S. Mahadeva Rao, Moses Rajasekara Pandian. Quercitrin a bioflavonoid improves the antioxidant status in streptozotocin: induced diabetic rat tissues. MOL CELL BIOCHEM 2011;358(1-2):121-129. https://doi.org/10.1007/s11010011-0927-x.

27. Ward AB, Mir H, Kapur N, Gales DN, Carriere PP, Singh S. Quercetin inhibits prostate cancer by attenuating cell survival and inhibiting anti-apoptotic pathways. WORLD J SURG ONCOL 2018;16(1). https://doi.org/10.1186/s12957-018-1400-z.

28. Cruz EA., Da-Silva SAG., Muzitano M F, Silva PM R, Costa SS, Rossi-Bergmann B. Immunomodulatory pretreatment with Kalanchoe pinnata extract and its quercitrin flavonoid effectively protects mice against fatal anaphylactic shock. INT IMMUNOPHARMACOL 2008;8(12):1616-1621. https://doi.org/10.1016/j.intimp.2008.07.006.

29. Kim JA, Jung YS, Kim MY, Yang SY, Lee S, Kim YH. Protective Effect of Components Isolated fromLindera erythrocarpa against Oxidative Stress-induced Apoptosis of H9c2 Cardiomyocytes. PHYTOTHER RES 2011;25(11):1612-1617. https://doi.org/10.1002/ptr.3465.

30. Xing LZ, Ni HJ, Wang YL. Quercitrin attenuates osteoporosis in ovariectomized rats by regulating mitogen-activated protein kinase (MAPK) signaling pathways. BIOMED PHARMACOTHER 2017;89:1136-1141. https://doi.org/10.1016/j.biopha.2017.02.073.

31. Wang T, Liu Q, Tjhioe W, Zhao JM, Lu AP, Zhang G, Tan RX, Zhou MY, Xu JK, Feng HT. Therapeutic Potential and Outlook of Alternative Medicine for Osteoporosis. CURR MEDICINE TARGETS 2017;18(9):1051. https://doi.org/10.2174/1389450118666170321105425.

32. Nowak B, Matuszewska A, Nikodem A, Filipiak J, Landwójtowicz M, Sadanowicz E, Jędrzejuk D, Rzeszutko M, Zduniak K, Piasecki T, Kowalski P, Dziewiszek W, Merwid-Ląd A, Trocha M, Sozański T, Kwiatkowska J, Bolanowski M, Szeląg A. Oral administration of kaempferol inhibits bone loss in rat model of ovariectomy-induced osteopenia. PHARMACOL REP 2017;69(5):1113-1119. https://doi.org/ 10.1016/j.pharep.2017.05.002. 
33. Wong SK, Chin KY, Ima-Nirwana S. The Osteoprotective Effects Of Kaempferol: The Evidence From In Vivo And In Vitro Studies. 2019;Volume 13:3497-3514. https://doi.org/ 10.2147/DDDT.S227738.

34. Weitzmann MN. Bone and the Immune System. TOXICOL PATHOL 2017;45(7):911-924. https://doi.org/10.1177/0192623317735316.

35. Zha L, He L, Liang YJ, Qin H, Yu B, Chang LL, Xue L. TNF-a contributes to postmenopausal osteoporosis by synergistically promoting RANKL-induced osteoclast formation. BIOMED PHARMACOTHER 2018;102:369-374. https://doi.org/10.1016/j.biopha.2018.03.080.

36. Wang T, He C. TNF-alpha and IL-6: The Link between Immune and Bone System. CURR MEDICINE TARGETS 2020;21(3):213-227. https://doi.org/10.2174/1389450120666190821161259.

37. Tong XS, Gu JH, Song RL, Wang D, Sun ZQ, Sui C, Zhang C, Liu XZ, Bian JC, Liu ZP. Osteoprotegerin inhibit osteoclast differentiation and bone resorption by enhancing autophagy via AMPK/mTOR/p70S6K signaling pathway in vitro. J CELL BIOCHEM 2018;120(2):1630-1642. https://doi.org/10.1002/jcb.27468.

38. Santen RJ, Simpson E. History of Estrogen: Its Purification, Structure, Synthesis, Biologic Actions, and Clinical Implications. ENDOCRINOLOGY 2019;160(3):605-625. https://doi.org/10.1210/en.201800529.

39. Wein MN, Kronenberg HM. Regulation of Bone Remodeling by Parathyroid Hormone. CSH PERSPECT MED 2018;8(8):a031237. https://doi.org/ 10.1101/cshperspect.a031237.

40. Hert MD, Detraux J, Stubbs B. Relationship between antipsychotic medication, serum prolactin levels and osteoporosis/osteoporotic fractures in patients with schizophrenia: a critical literature review. EXPERT OPIN MEDICINE SAF 2016;15(6):809-823. https://doi.org/10.1517/14740338.2016.1167873.

41. Melville KM, Kelly NH, Khan SA, Schimenti JC, Ross FP, Main RP, Meulen MCH. Female Mice Lacking Estrogen Receptor-Alpha in Osteoblasts Have Compromised Bone Mass and Strength. J BONE MINER RES 2014;29(2):370-379. https://doi.org/10.1002/jbmr.2082.

42. Moriarty K, Kim KH, Bender JR. Estrogen Receptor-Mediated Rapid Signaling. ENDOCRINOLOGY 2006;147(12):5557-5563. https:// doi.org/ 10.1210/en.2006-0729.

43. Sharma AR, Nam JS. Kaempferol stimulates WNT/ $\beta$-catenin signaling pathway to induce differentiation of osteoblasts. J Nutri Biochem 2019;74:108228. https://doi.org/10.1016/j.jnutbio.2019.108228.

44. Du D, Z. Zhou, L. Zhu, X. Hu, J. Lu, C. Shi, F. Chen, A. Chen. TNF-alpha suppresses osteogenic differentiation of MSCs by accelerating P2Y2 receptor in estrogen-deficiency induced osteoporosis. BONE 2018;117:161-170. https://doi.org/10.1016/j.bone.2018.09.012.

45. Shahzad H, Giribabu N, Muniandy S, Salleh N. Quercetin induces morphological and proliferative changes of rat's uteri under estrogen and progesterone influences. Int J Clin Exp Pathol. 2014 Aug 15;7(9):5484-94. PMID: 25337190; PMCID: PMC4203161.

46. Yuan Z, Min J, Zhao Y, Cheng Q, Wang K, Lin S, Luo J, Liu H. Quercetin rescued TNF-alpha-induced impairments in bone marrow-derived mesenchymal stem cell osteogenesis and improved 
osteoporosis in rats. Am J Transl Res. 2018 Dec 15;10(12):4313-4321. PMID: 30662673; PMCID: PMC6325508.

\section{Figures}

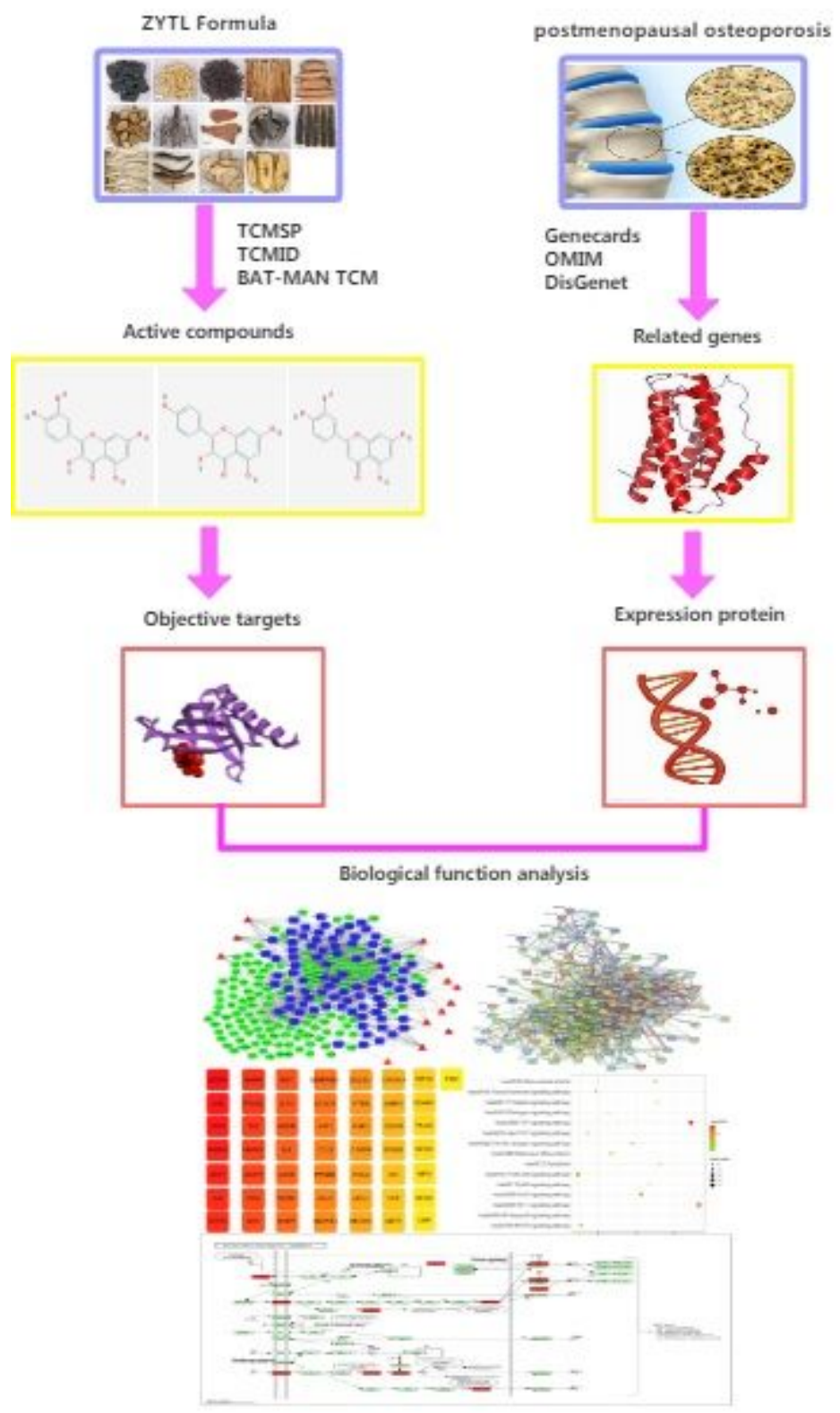

\section{Figure 1}

Flowchart showing the network pharmacology approach for determining the molecular mechanisms of Ziyin Tongluo Formula (ZYTLF) against postmenopausal osteoporosis (PMOP) and molecular docking technology for verifying it. 

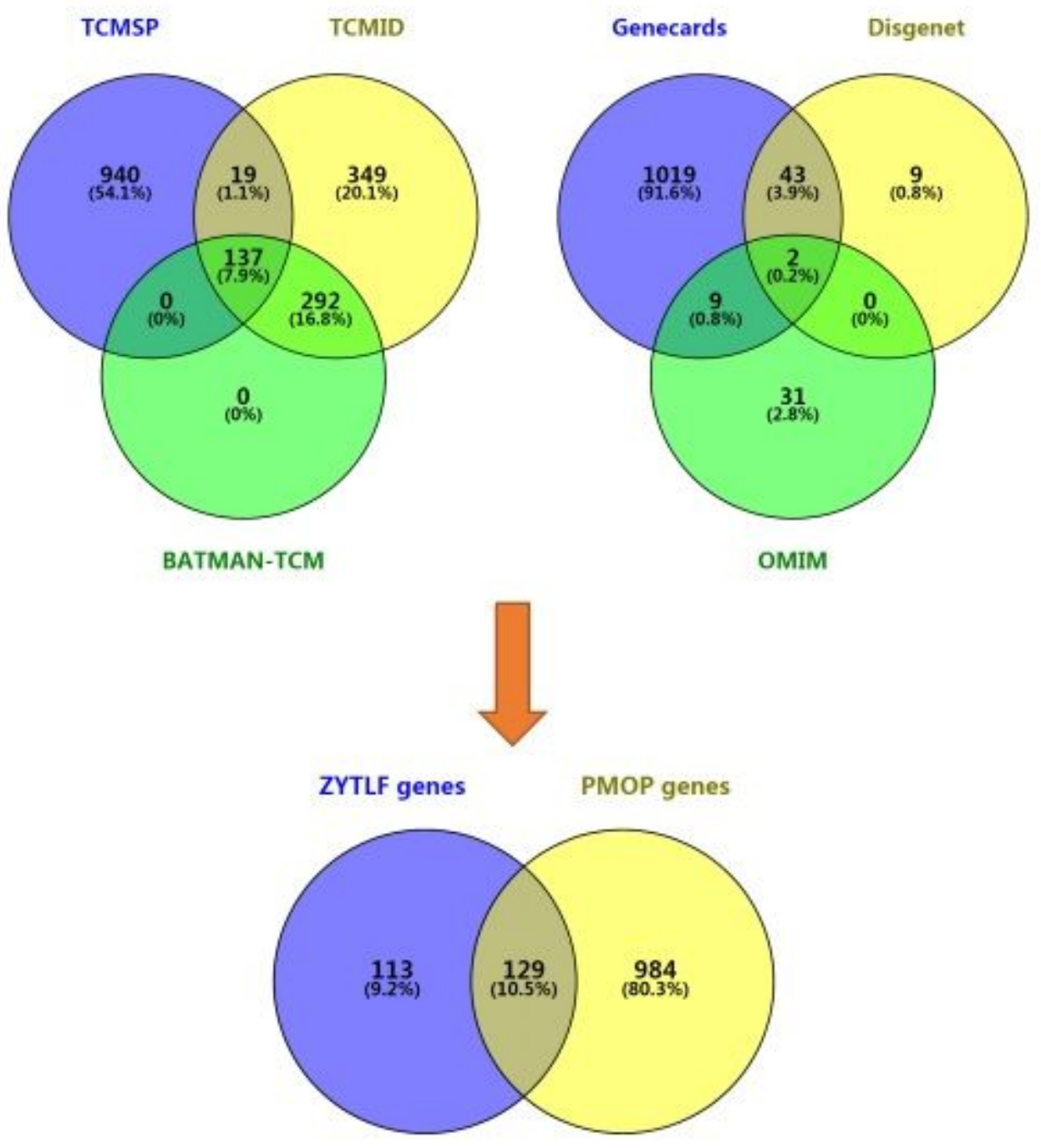

Figure 2

There are 242 active compounds of ZYTLF and 1113 genes associated with PMOP. 129 overlapping genes were selected as potential targets for further study. 


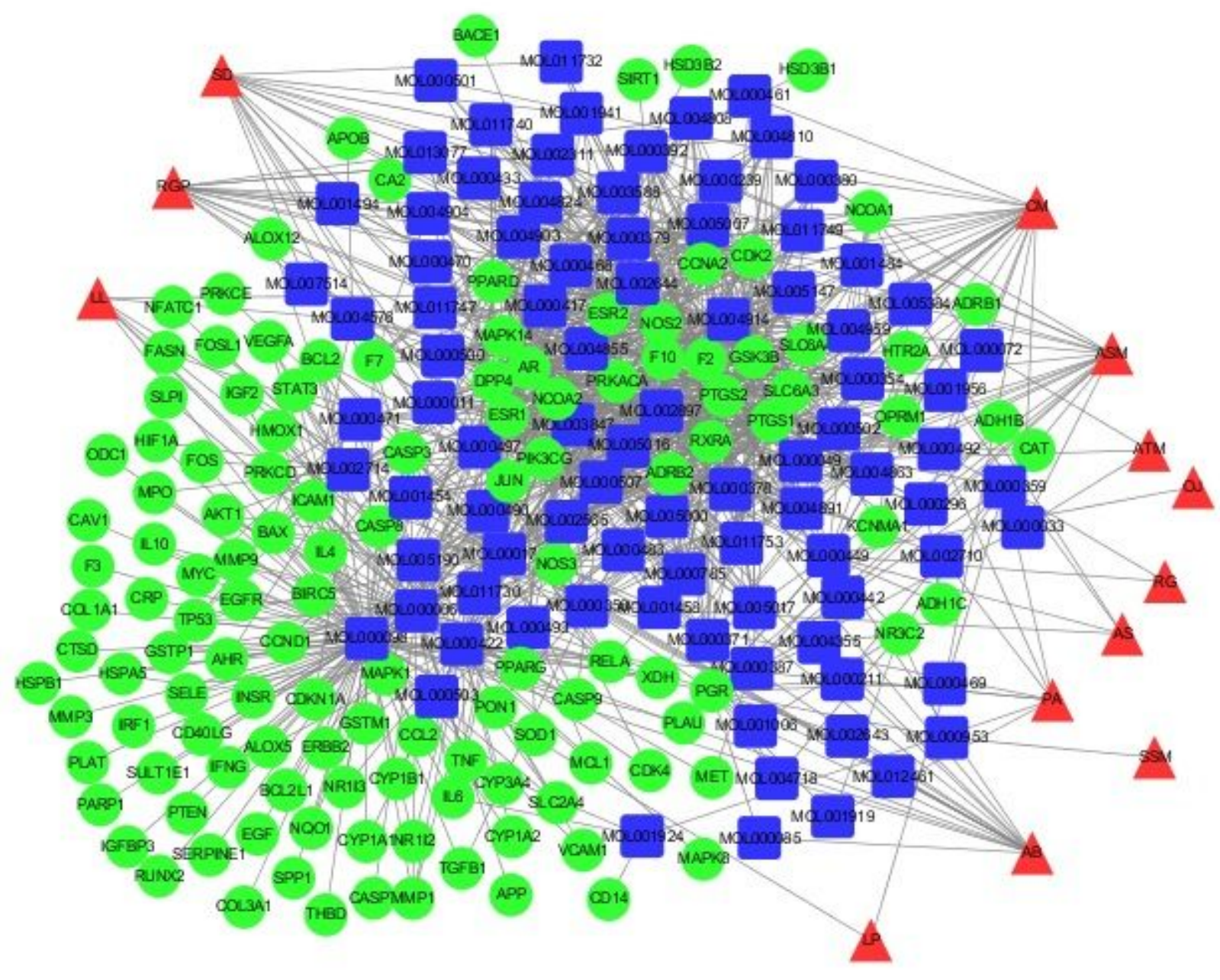

Figure 3

herb-active ingredient-overlapping gene" network The red triangle in the network represent traditional Chinese herbs, blue square represent active ingredients, and green circle represent overlapping target genes. Lines represent relationship between nodes. 


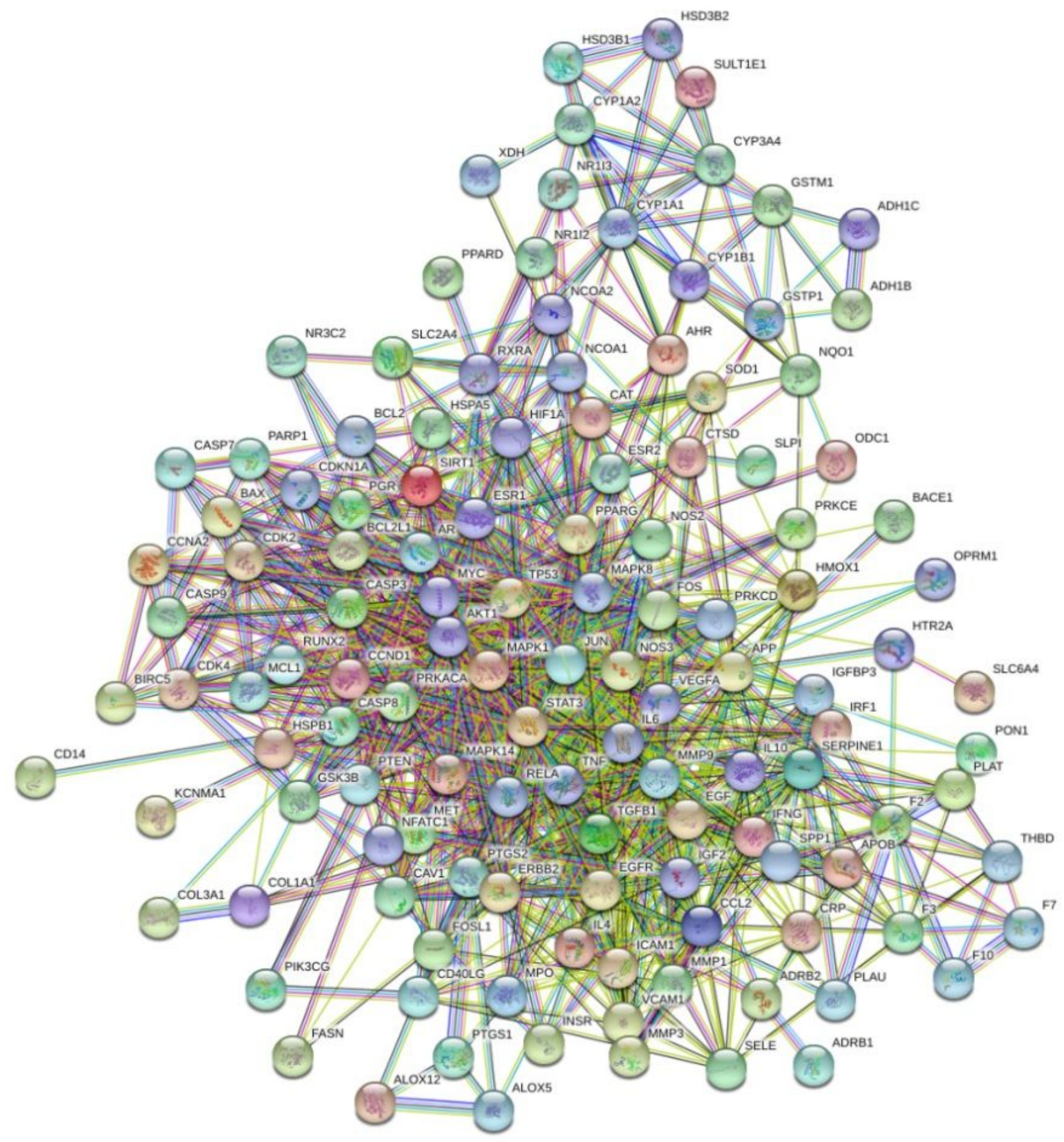

Figure 4

overlapping gene protein-protein interaction network diagram In the picture, the nodes represent proteins, and the lines represent the interactions between proteins. 


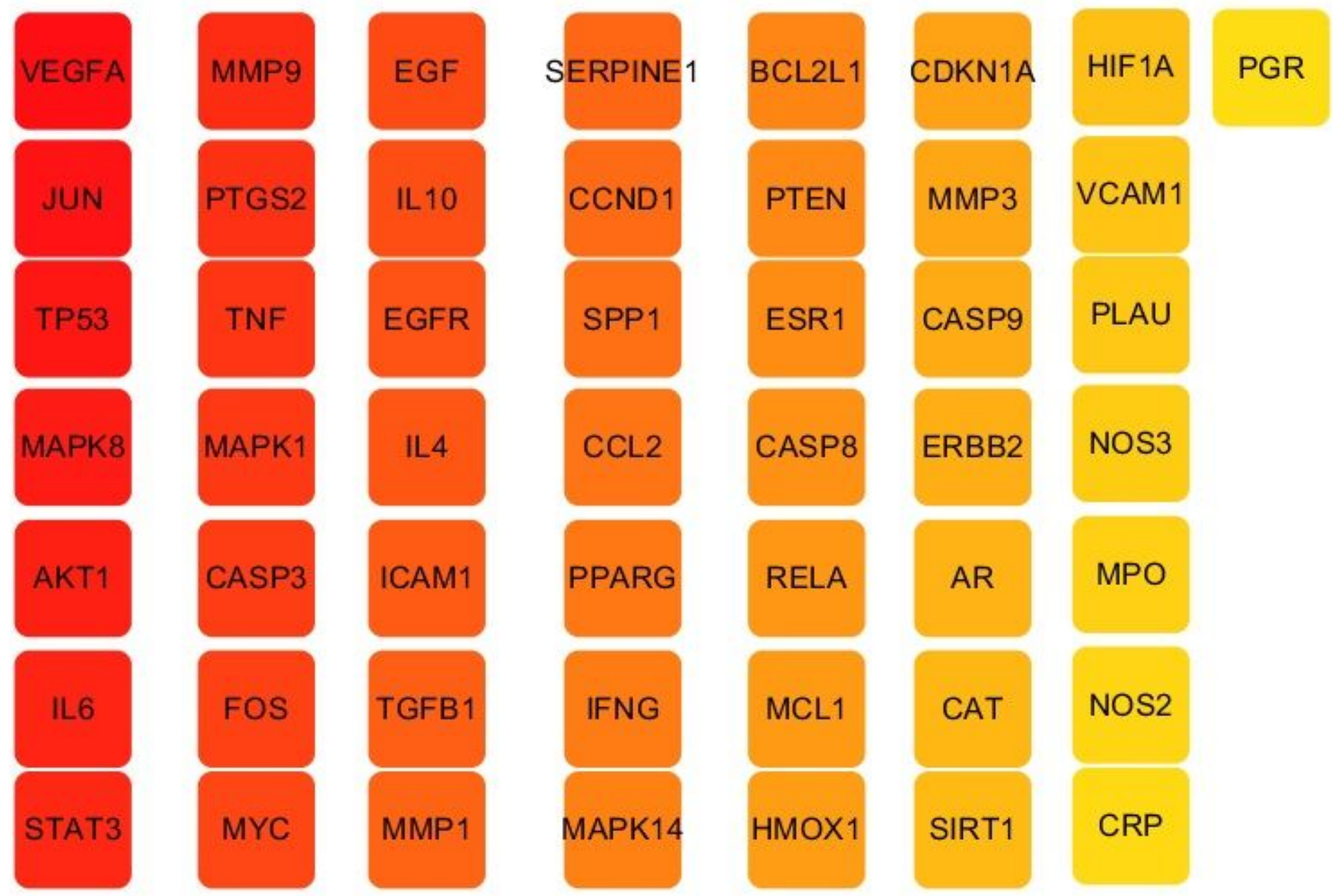

Figure 5

Key genes in overlapping target genes The color of the node changed from light yellow to dark red, indicating that the higher the MCC value was, the more significant the role it played in the network. 
a

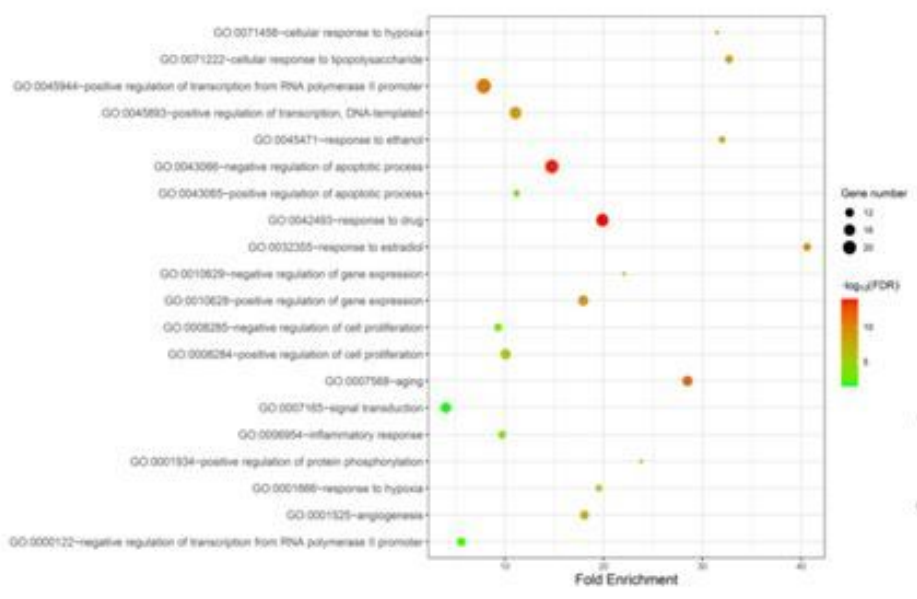

C

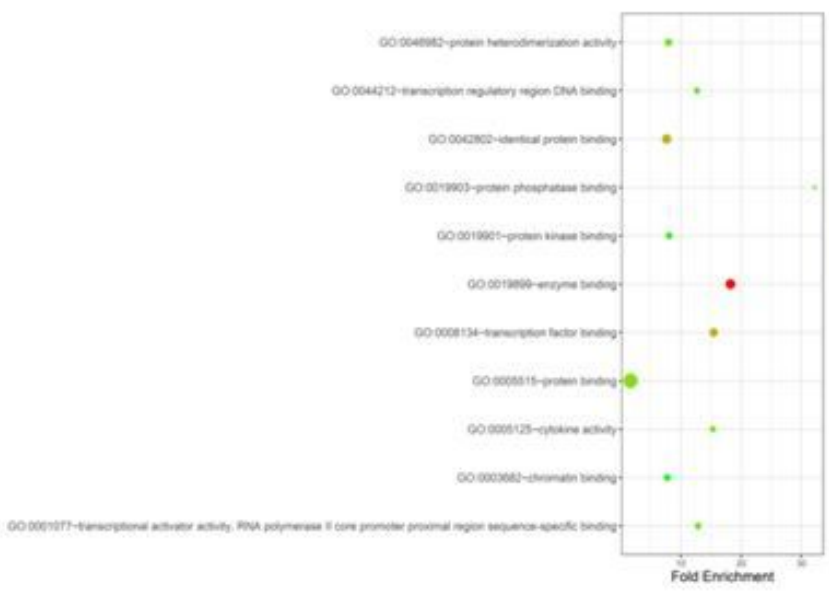

$\mathrm{b}$

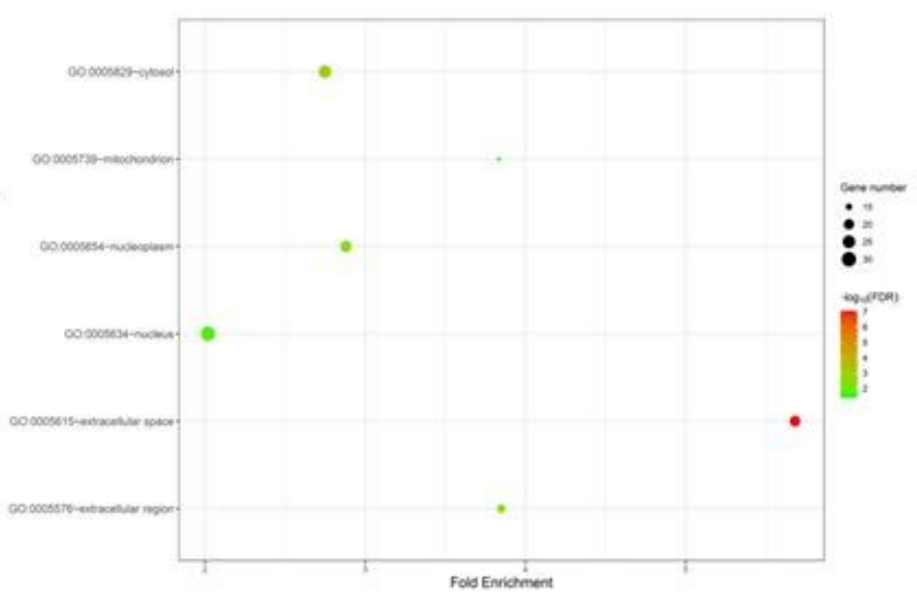

$\mathrm{d}$

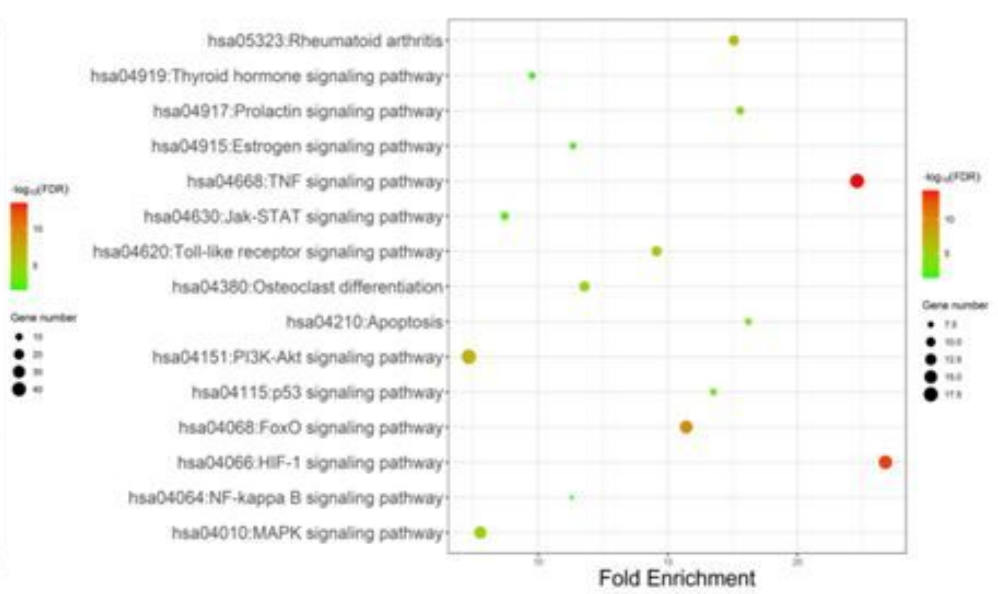

\section{Figure 6}

GO analysis and KEGG pathway enrichment analysis of Key gene In the bubble diagrams above (a-d), the ordinate represents the names of BP, CC, MF terms and pathways, respectively, and the abscissa represents the degree of enrichment. The smaller the FDR is, the higher the importance of enrichment, and the redder the color on the diagram. 


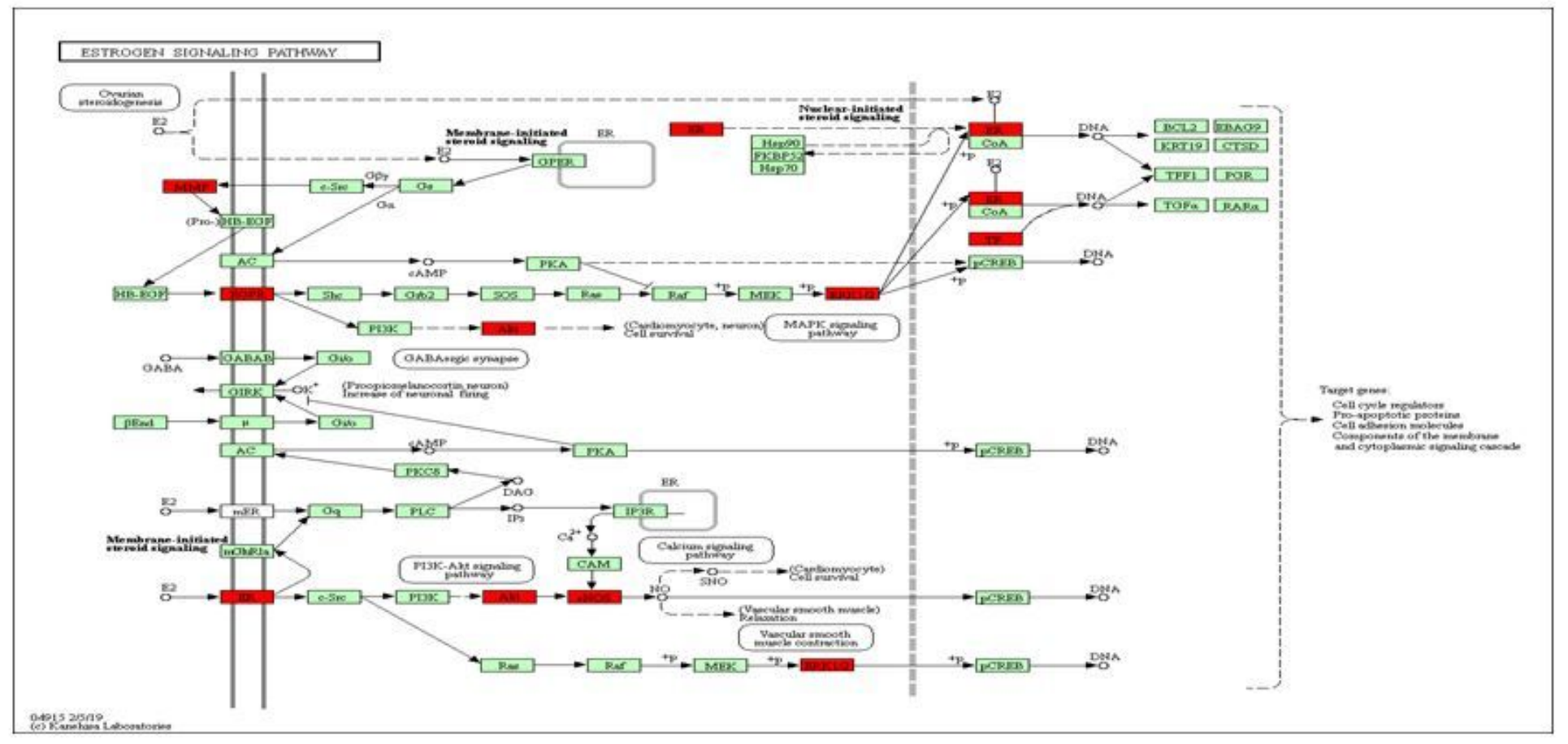

Figure 7

Specific mechanism of key gene enrichment in estrogen signaling pathway

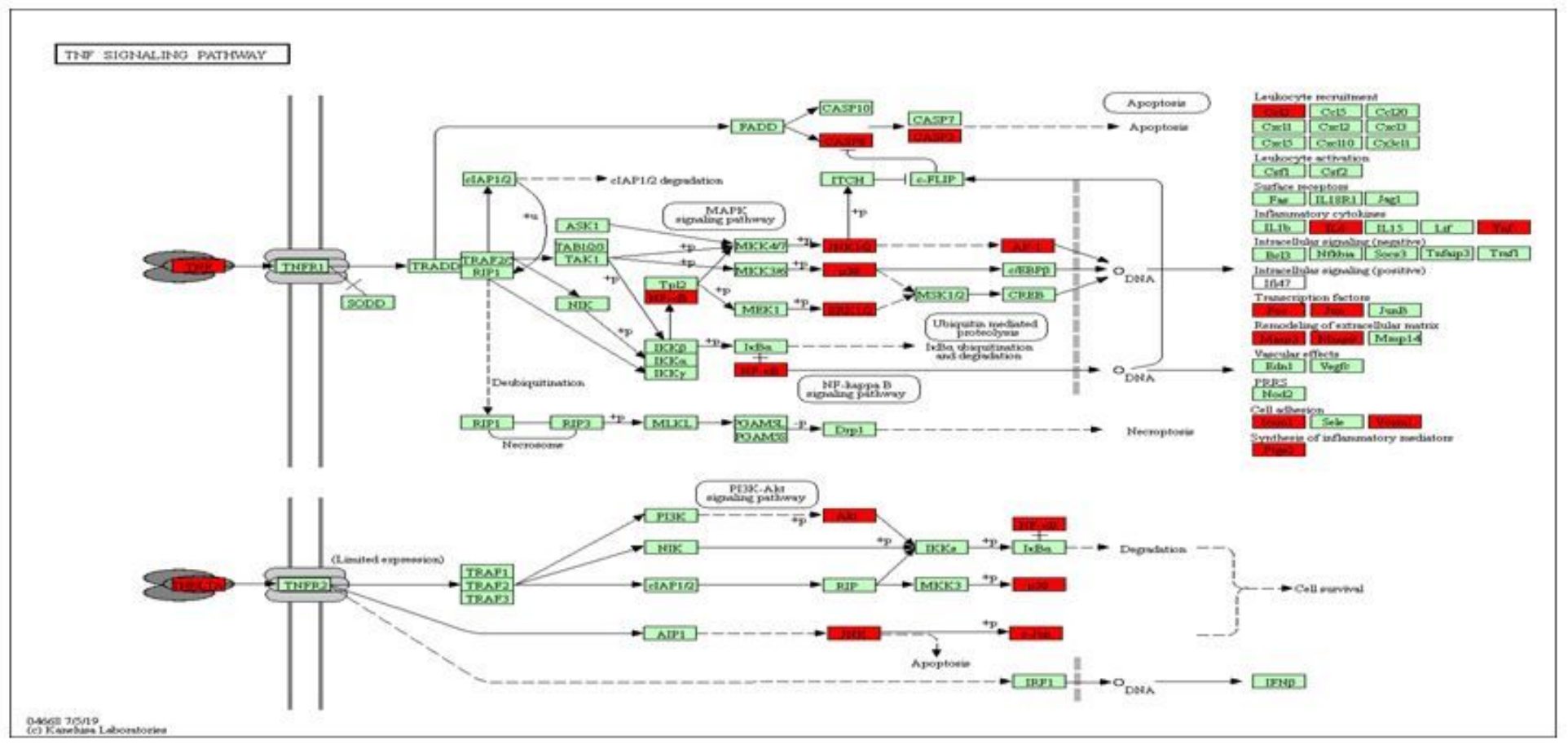

\section{Figure 8}

Specific mechanism of key gene enrichment in TNF signaling pathway 

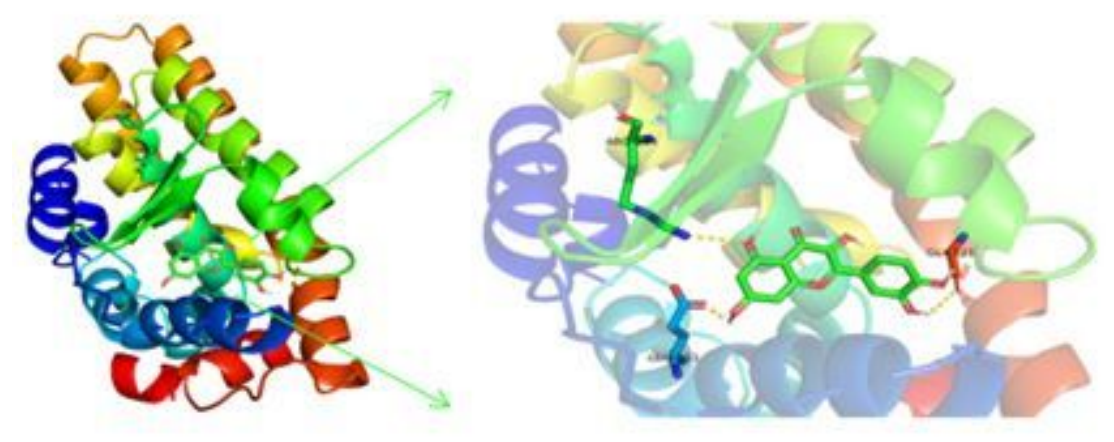

Figure 9

ESR1 protein - quercetin
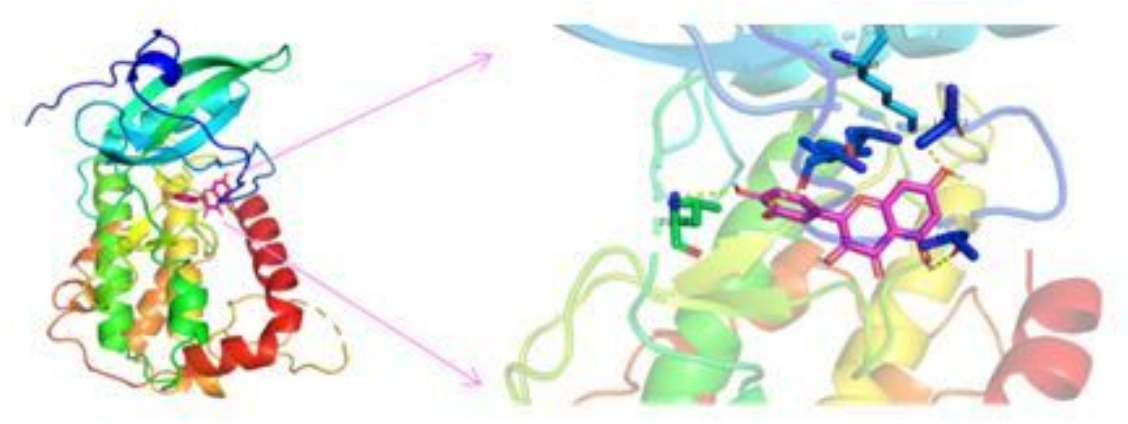

Figure 10

TNF protein - quercetin

\section{Supplementary Files}

This is a list of supplementary files associated with this preprint. Click to download.

- Additionalfile2Tables2.xls

- Additionalfile1Tables1.xls

- Additionalfile4Tables4.xls

- Additionalfile5Tables5.xls

- Additionalfile3TableS3.xls

- formula.docx 